\title{
Autologous Osteoblast-cell Therapy in Orthodontics and Implantology: A Single-center Experience of Nine Patients
}

\author{
Dilip Deshpande
}

\begin{abstract}
Resorption of bone post tooth extraction is an established fact. In addition to this, pneumatization of maxillary sinuses further reduced the available bone and space for dental implant restoration. Traumatic loss of bone and bone loss due to cystic disease adds another dimension to clinical presentation and management. Various modalities have been used for bone replacement or restoration and are well documented. Here, we present a case series of nine patients, wherein a novel autologous cultured osteoblast cell product was used for bone augmentation in different clinical settings, with a quite satisfactory long-term outcome. We recommend the use of this revolutionary approach in rightly selected patients for permanent bone regeneration in the treatment of various oral and maxillofacial defects.
\end{abstract}

Keywords: Autologous osteoblast-cell therapy, Implant, Orthodontistry.

International Journal of Oral Implantology and Clinical Research (2018): 10.5005/jp-journals-10012-1184

\section{INTRODUCTION}

Edentulous bone loss is very common and bone and alveolar ridge augmentation has been a prerequisite for dental implants. ${ }^{1}$ Many different clinical conditions like atrophy, periodontal disease, and consequence of trauma result in unfavorable alveolar ridge. This leads to insufficient bone volume and intramaxillary spatial discordance making dental implantation almost impossible. ${ }^{2}$ Involvement of maxillary sinuses in such oral and maxillofacial pathologies cannot be ignored. When teeth are lost, the sinus expands in the inferior region at the expense of surrounding bone. ${ }^{3}$ Pneumatization or enlargement of the sinus limits the quantity of alveolar bone available for implant and may result in compromised osseointegration of newly formed bone and stability of implants. ${ }^{4}$

It has been reported that reductions in the residual alveolar ridge are most prominent within 6 months of the tooth extraction, and continue at a slower rate thereafter. ${ }^{5}$ Bone augmentation, as reported by one group, is required in as many as $50 \%$ of the patients undergoing procedures for dental implantations. ${ }^{6}$ Requirement of bone tissue, the area, height, and total volume to be achieved are factors to be considered before choosing the bone reconstruction or regeneration modality. The goals of osseous replacement are maintenance of contour, elimination of dead space, and reduce postoperative infection, thus, enhancing bony and soft-tissue healing. ${ }^{7}$ Recent progress in regenerative approaches has conferred marked benefits in prosthetic dentistry, enabling regeneration of the atrophic alveolar ridge. Various bone-grafting options are available and are being used over decades. Physiologically, the bone grafting should holistically aim at achieving osteoinduction, osteoconduction, and osteogenesis that collectively would result in the formation of vascularized bone and complete osseousintegration. ${ }^{8}$ Finally, functional as well as esthetic restoration of lost teeth and face are very crucial.

Among various options available for bone augmentation allografts with or without membranes, scaffolds and/or titanium mesh have given a good long-term outcome so far. Chiapasco et al. $^{2}$ in their extensive review article elaborately described and analyzed the pros and cons of various bone augmentation procedures. Osteoinductive effect of growth factors like bone morphogenic proteins is still in the experimental phase and
Department of Dentistry, Lilavati Hospital and Research Centre Mumbai, Maharashtra, India

Corresponding Author: Dilip Deshpande, Department of Dentistry, Lilavati Hospital and Research Centre, Mumbai, Maharashtra, India, Phone: +91 22-2446 7446, e-mail: drddeshpande123@gmail.com

How to cite this article: Deshpande D. Autologous Osteoblastcell Therapy in Orthodontics and Implantology: A Single-center Experience of Nine Patients. Int J Oral Implantol Clin Res 2018;9(1-3): 55-59.

Source of support: I acknowledge Regrow Biosciences Pvt Ltd for providing the study drug in form of autologous cultured osteoblast cells for all 9 patients

\section{Conflict of interest: None}

has extremely limited applications. In-lay or on-lay bone grafts, guided bone regeneration (GBR), split-ridge/ridge expansion techniques, and alveolar distraction osteogenesis represent commonly used methods to recreate correct intermaxillary relationships, adequate bone morphology, and the volume required for implant placement. ${ }^{9}$ This group concluded that it is difficult or impossible to determine that one surgical procedure offers better outcome than another, as far as predictability of the augmentation and survival/success rates of implants placed in the augmented sites is concerned. Later, Sakkas et al. ${ }^{10}$ in their study of 279 patients and 456 evaluable autologous augmentation procedures reported that $95.6 \%$ of grafts healed successfully (436 out of 456). They reported autologous bone grafts to be "gold standard" for alveolar bone augmentation.

The true regenerative approaches involve the use of various stem cell sources, scaffolds through tissue engineering, and, going forward, genetically programmed cells. In an overview, Sobitha et al. proposed that clinical use of mesenchymal stem cells for bone regeneration should overcome the limitations of other grafts. ${ }^{11}$ They also mention that tissue engineering would have much larger scope and can provide numerous clinical applications that may even include regrowing of the lost tooth. According to Masaru et al., current consensus on cell-based bone augmentation therapies emphasizes on cell sources, transplanted cell survival, endogenous 
stem cell recruitment, and immunomodulatory function of transplanted progenitor cells. ${ }^{12}$

Sporadic case reports have been published so far where mesenchymal stem cells (MSCs) from various sources have been used along with other bone-grafting materials in alveolar bone regeneration and maxillary sinus lift. ${ }^{13-15}$ They have individually reported bone regeneration to about $33-41 \%$ in 1-6 patients.

\section{Our Hypothesis}

Although our 30 years of clinical experience reiterate that the space available in the oral and the maxillary region is variable, attaining precise thickness or thinness, height, length, and breadth within the cavity with newly restored bone graft is difficult to achieve. Among the biological or regenerative approaches, we have some experience of using platelet-rich fraction (PRF); but the results have not been consistent and long lasting. The use of membranes and scaffolds that are flexible and pliable and titanium mesh-like hard supports gave better results. Having understood the theoretical approach of the use of MSCs with the absence of satisfactory clinical evidence, we were not very much convinced to use that modality. But, intrinsic augmentation of bone regeneration using autologous cultured osteoblast cells, proposed to us, was truly a novel concept. This osteoblast product provided in a unique patented delivery system was thought to be able to occupy every possible niche and help body's bone remodeling to regenerate the required bone with complete osteoinductive and osteoconductive properties, thereby achieving osseousintegration of the regenerated bone.

Our group used autologous cultured osteoblast cell product, along with other conventional augmentation techniques as and when required for a series of nine patients with various pathological clinical presentations.

\section{Materials and Methods}

We present herewith our clinical experience over 5 years of treating nine patients with varied age groups and history using autologous live osteoblast cells cultured from patients' bone marrow along with bone-grafting material as and when required.

The investigations required for oral-maxillo-facial and dental implantology domain were restricted to orthopantomogram (OPG) (which is an advanced digital X-ray technique that captures and displays all teeth of upper and lower jaw on a single film) and conebeam-computed tomography (CBCT) (a medical imaging technique consisting of X-ray computed tomography where the X-rays are divergent, forming a cone). In all patients, histopathological assessment was done before the procedure and at a time suitable post-osteoblast implant.

For all the patients, bone marrow biopsy was picked up in a hospital outpatient setting and, for each patient, the cultured osteoblast cells were made available within 4-5 weeks of the biopsy.

Our aim was to observe the time required for bone reconstruction and/or augmentation so as to make the ridge ready for the dental implantation, and, later, the survival of the newly regenerated bone, and thereby implants.

We present the detailed patient data herewith.

\section{Case Description}

Patient selection was based on age (from 18 years to 65 years) and clinical presentation indicating edentulous bone loss with or without pneumatization. Depending on the site of defect, sinus lift was added in the surgical procedure.

Of the nine patients treated, there were two females and seven males. Maxillary and/or mandibular reconstruction, ${ }^{6}$ mandibular cystic disease, ${ }^{1}$ traumatic loss of tooth, ${ }^{1}$ and teeth and bone loss due to severe periodontal disease ${ }^{1}$ were clinical presentations and reasons for a need of bone augmentation. Sinus lift was performed in two patients.

\section{CASE 1}

A female patient, a scientist by profession, 63-year old. The patient's clinical history was uncomfortable denture fitting due to the absence of bone support, with increasing bone loss with time. Symptoms started at the age of 37 with the shaking of bone plates accompanied by inadvertent tongue and cheek biting. The speech was abnormal. This resulted in the removal of teeth and fitting of dentures. The patient presented to our clinic when she had stopped using the dentures for 4-5 years. CBCT and OPG were used for the investigation. She had very scanty bone in the anterior, posterior, as well as vertical zone. Treatment regimen involved autologous cultured osteoblast implant and cortical bone chips for maxillary reconstruction (Figs 1 to 3 ).

\section{CASE 2}

The patient was an 18-year-old male student. The patient suffered the posttraumatic loss of a tooth with the secondary infection 2 years ago. The bone loss was evident on CBCT and OPG investigations that were done after the patient presented at the clinic. Treatment regimen involved autologous osteoblast implant and resorbable membrane as a scaffold.

\section{Case 3}

The patient was a 44-year-old housewife. The case presented was a loss of major maxillary bone. The patient presented with advanced periodontal disease, with loss of complete maxillary teeth except the canine region. There was bilateral pneumatization in the maxillary sinuses. Investigations conducted were CBCT and OPG. Treatment administered was autologous osteoblast implant

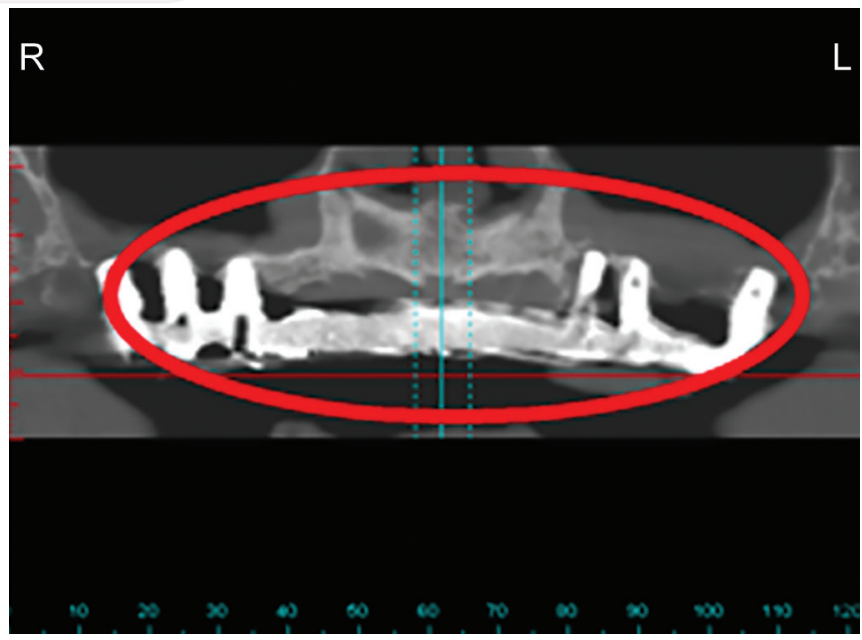

Fig. 1: OPG images showing preoperative, near complete absence of maxillary bone plate 

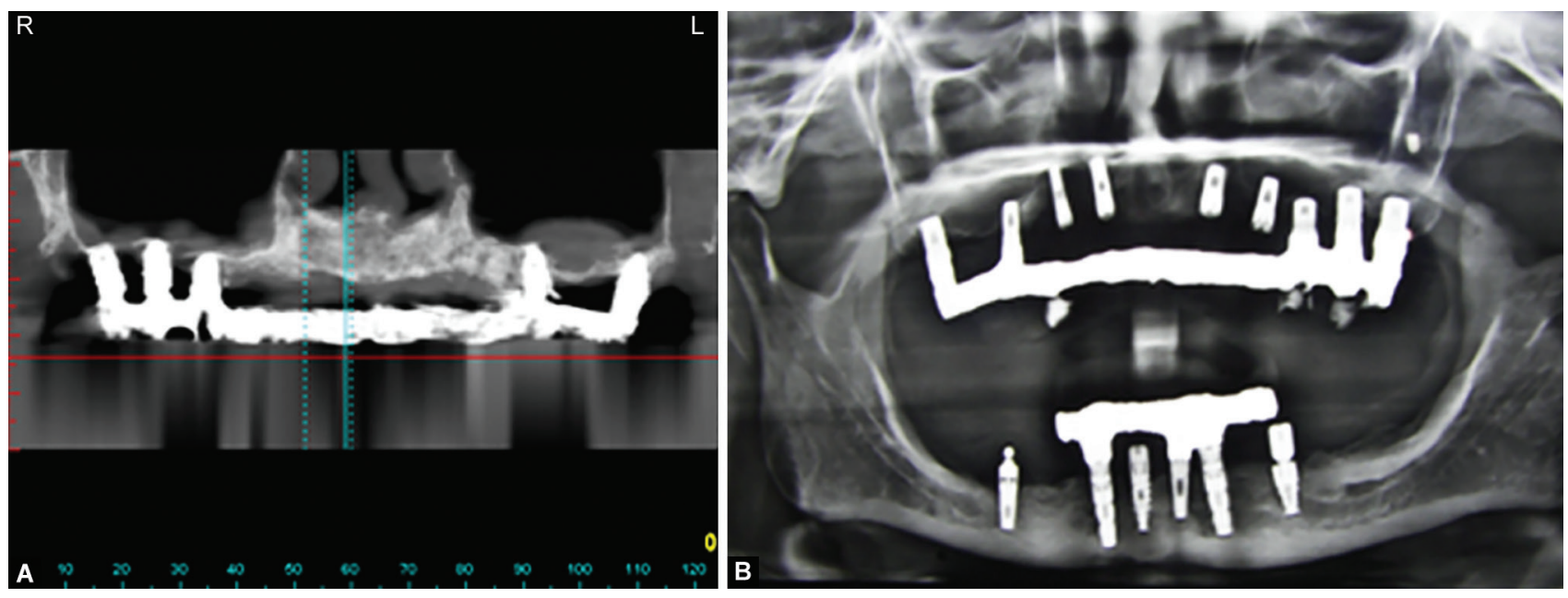

Figs 2A and B: (A) OPG images showing regenerated bone postoperative at 3 months; (B) Postoperative at 4 months with dental implants

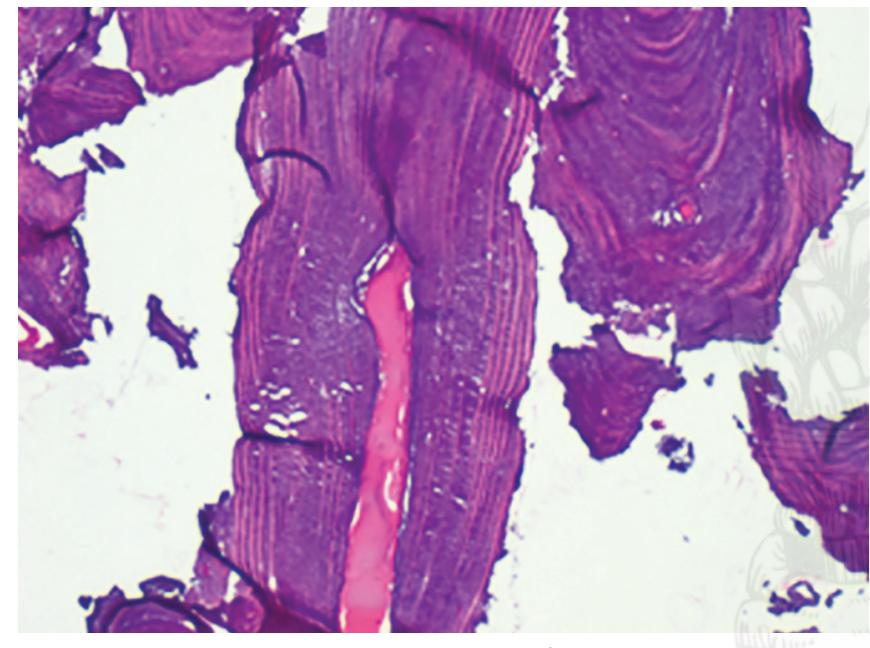

Fig. 3: Dense cortical bone with evidence of remodeling

and cortical bone chips (from iliac crest) + bilateral direct sinus lift (Figs 4 to 6).

\section{CASE 4}

The patient was a 42-year old and presented with alveolar ridge defect in the mandibular anterior region. It was a case of traumatic loss of vertical as well as horizontal loss of alveolar bone. Investigations conducted after the presentation to the clinic were OPG and CBCT. The treatment regimen was autologous osteoblast implant on an autograft with a titanium mesh support system (Fig. 7).

\section{CASE 5}

The fifth patient, a 26-year-old male, presented with a mandibular defect on the posterior left side. The history revealed accidental fall resulting in mal-united fracture of the mandible. CBCT and OPG were conducted that revealed major bone loss. Treatment included autologous osteoblast implant over cortical bone graft from iliac crest and titanium mesh support.

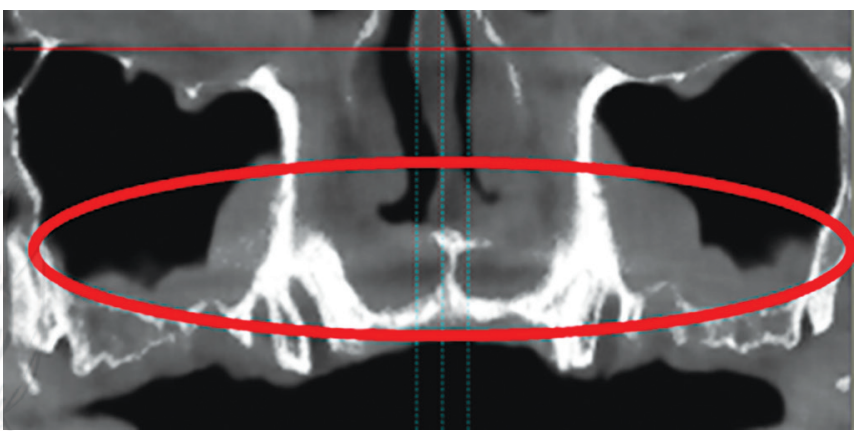

Fig. 4: OPG preoperative panoramic view showing severe maxillary bone atrophy

\section{CASE 6}

A 55-year-old male reported with abnormal facial features and difficulty in chewing and biting. The primary investigations revealed that this deformity was due to major bone loss in the mandibular region. Patient's history could not decipher any specific reason responsible for this pathology. The patient was counseled and then treated using autologous osteoblast implant over bone graft along with sinus lift.

\section{CASE 7}

The seventh case was of a 32-year-old male patient. The patient presented with difficulty while talking with pain when the pallet was touched. The pallet was palpable and very tender. Eating actions were also complicated. Investigations done via OPG showed the presence of two dentigerous cysts adjacent to each other, within the maxillary pallet on the left side amounting to $2.5 \times 3.5 \times 1 \mathrm{~cm}^{3}$ space being occupied. Treatment involved cyst excision followed by artificial crushed bone overlaid with autologous osteoblast implant.

\section{CASE 8}

This was a 43-year-old male presented with pneumatized bilateral sinuses. The patient had a history of persistent tobacco chewing. The CBCT and OPG were conducted after the patient was presented 

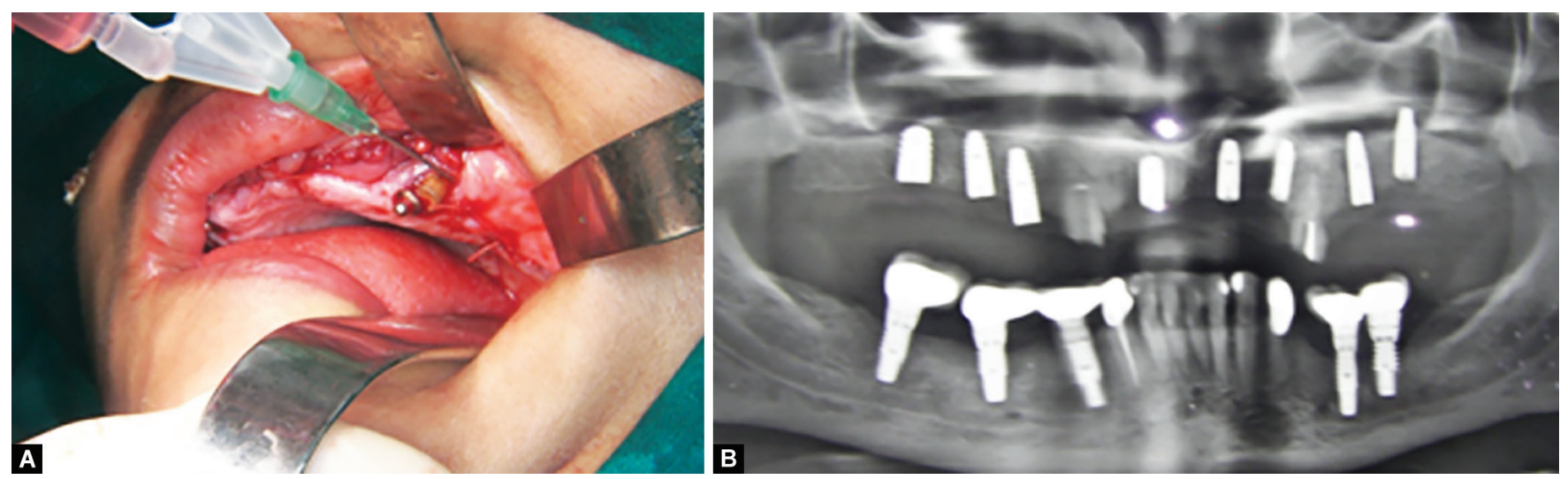

Figs 5A and B: (A) Sinus lift and autologous cultured osteoblast implant; (B) Postoperative at 4 months with dental implants

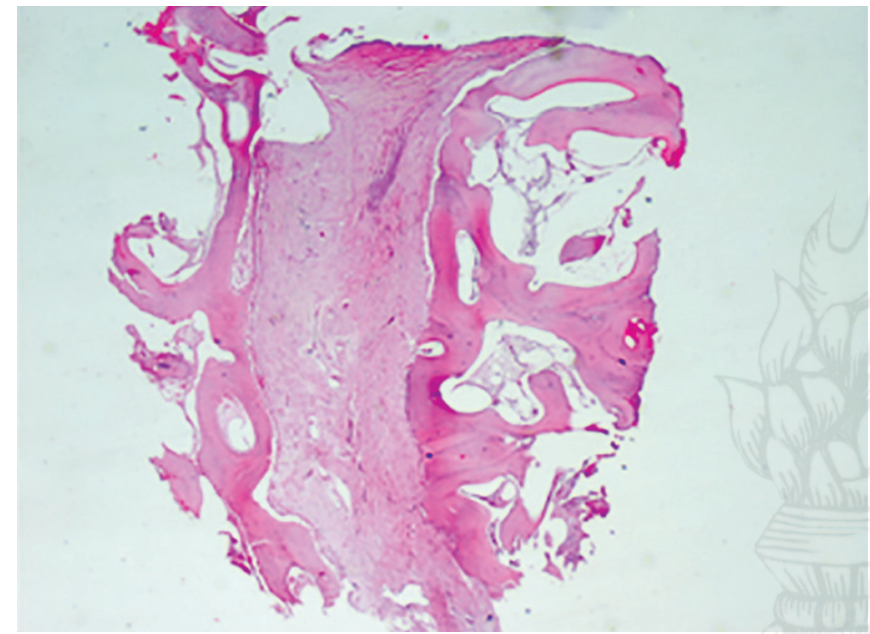

Fig. 6: Fibrovascular marrow space

at the clinic. It revealed the right-side defect to be $14 \times 24 \times 14 \mathrm{~mm}^{3}$ and the left-side defect to be $14 \times 14 \times 15 \mathrm{~mm}^{3}$. Treatment included bone graft with autologous osteoblast implant, bilaterally.

\section{CASE 9}

The patient involved was a 51-year-old Indian male. The patient presented with left maxillary teeth loss, possibly due to some unidentified defects. Diagnosis showed a defect in the canine region. OPG and $C B C T$ were done to locate the defects. Treatment for this patient involved sinus elevation $\left(14 \times 14 \times 12 \mathrm{~mm}^{3}\right)$ with the canine region vertical lift $\left(20 \times 14 \times 5 \mathrm{~mm}^{3}\right)$ using graft scaffold, autologous osteoblast implant with titanium mesh support for vertical reconstruction.

\section{Results}

After the bone augmentation procedure with autologous osteoblast implant and other respective adjuncts, all patients were followed up at a month's interval. The bone growth was assessed in clinical examination and at the end of third month, OPG was done. Once sufficient osseous growth, strong enough to have implant fixed was achieved, the dental implant procedure was performed. A few patients followed by regularly post-implant, while a few lost to follow-up, and a couple of available on telephone for follow-up.
The trephine bone sample was assessed by histopathology to ascertain the morphology.

In patient 1,6 months post-osteoblast-implant, dental implants could be placed in the anterior esthetic zone as enough bone volume was achieved. It was possible to give acceptable esthetic restoration. The trephine biopsy showed evidence of remodeling with vascularization.

Patient 3 had a good quality of bone within 3 months throughout the anterior and posterior maxilla; and eight dental implants were placed comfortably. Core biopsy in this patient showed regenerated bone with fibrovascular marrow spaces, and particulate auto-bone fully integrated with new bone.

In patient 4, it took about 2.6 months to achieve new bone with $4 \mathrm{~mm}$ width. The vertical height enough to place two implants was also achieved. New lamellar bone with remodeling lines was evident on the biopsy.

The patient 5 who had malunited fracture showed good union of fracture and implants could be placed in the posterior region.

In patient 7, after cyst removal, the new bone augmentation was $1.5 \mathrm{~cm}$ in height; $2.5 \mathrm{~cm}$ in length, and $2.5 \mathrm{~cm}$ in width. The defect was completely filled with new bone.

In patient 9, at 6 months postosteoblast treatment, $4.5 \mathrm{~cm}$ of vertical bone with $1 \mathrm{~cm}$ height was achieved. Dental implants were done comfortably.

For all patients, the dental implantation could be done from 3 months postosteoblast-implant procedure. The longest time gap was of 6 months, only for patient 1.

All patients resumed normal eating, biting, and chewing activities within about 1 month postdental implants. No patient had any other inadvertent intraoperative injury or nerve damage. Patients who also suffered from speech difficulty resumed to normal speaking. The longest follow-up was for 7 years postautologous cultured osteoblast treatment; and the entire dental implant is still intact in patient I. All other patients who were followed for between 6 months and about 3 years showed intact, uncompromised bone with dental implants fully functional. The patient with cystic disease did not have any recurrence of oral/dentigerous cyst. Patients who had pneumatization of sinuses also did not show any recurrence during the follow-up period.

Overall, all nine treated patients could achieve new bone in short time for dental implantation; and the survival of newly augmented bone and, hence, dental implants is as good as permanent, with no patient required to do any repeat procedure. 

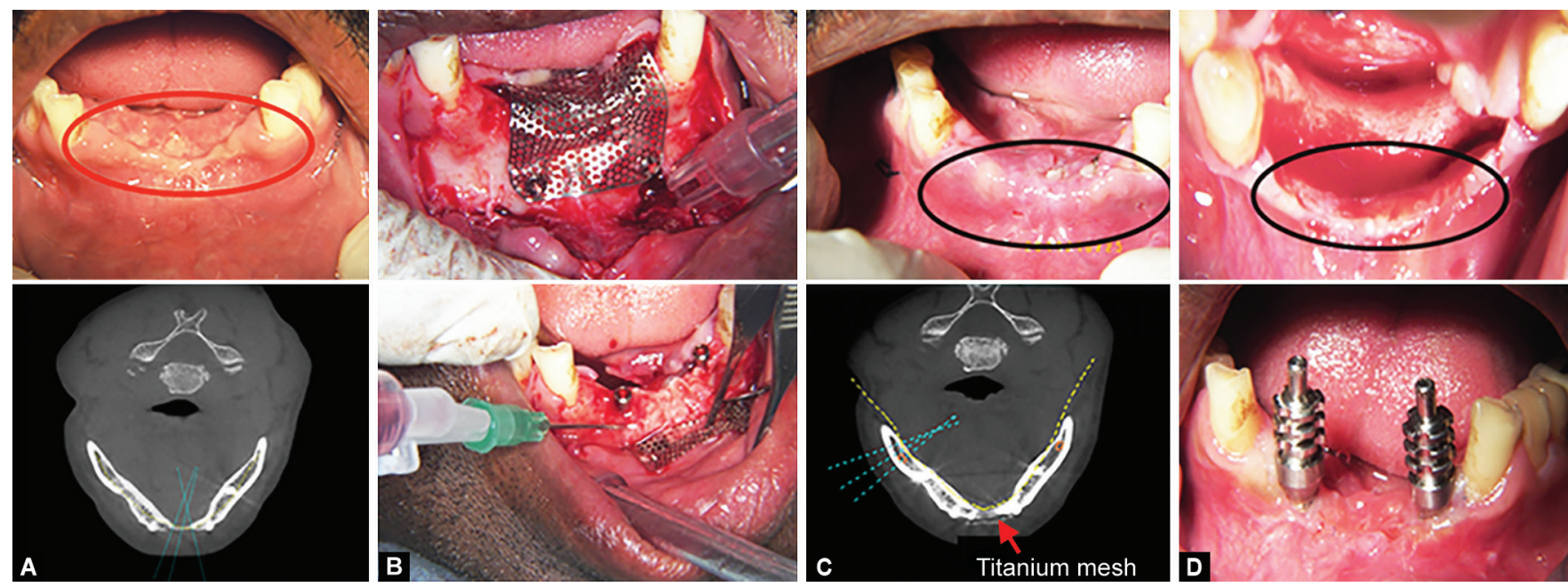

Figs 7A to D: (A) CBCT images and photographs showing preoperative, mandibular defect showing the absence of bone; (B) The use of titanium mesh and autologous cultured osteoblast implant; (C) Condition post-autologous cultured osteoblast treatment, 3 months. Ridge shows bone growth giving height to the mandible; (D) Post 6 months after treatment, dental implants were done

\section{Discussion}

There has been a lot of postulation and hypotheses on and about the possibility of stem cell and/or cell-based therapy for various oral and maxillofacial conditions. While actual therapeutic action of MSCs from any source is still a mystery, our experience of using autologous cultured osteoblast cells has been very encouraging. The histopathological assessment also has proved that the new regenerated bone is like the native has enough vascularization and lamellar growth. The newly formed bone completely integrates with the native bone and offers room and strength for the dental implants to be placed. The long-term follow-up, with the dental implants remaining intact for up to 7 years, also has ensured that the newly regenerated bone undergoes normal remodeling without abnormal or excessive resorption.

\section{Conclusion}

Among all the bone augmentation options available, thus, far, autologous, cultured osteoblast cells have given best results based on the flexibility of use with other adjuncts, quality of bone regenerated, and its structural and functional suitability. Our center would be pioneer in reporting the use of autologous cultured osteoblast cells in a series of nine patients, with follow-up of as long as 7 years. We recommend the use of this novel cell-based therapy approach in rightly selected patients.

\section{References}

1. Albrektsson T, Zarb G, et al. The long term efficacy of currently used dental implants: a review and proposed criteria of success. Int J Oral Maxillofac Implants 1986;1:11-25.

2. Chiapasco M, Casentini P, et al. Bone augmentation procedures in implant dentistry. Int J Oral Maxillofac Implants 2009;24(Suppl): 237-259.
3. Misch CE. Maxillary sinus augmentation for endosteal implants: organized alternative treatment plans. Int J Oral Implantol 1987;4: 49-58.

4. Tatum H. Maxillary and sinus implant reconstructions. Dent Clin North Am 1986;30:207-229.

5. Jahangiri $L$, Devlin $H$, et al. Current perspective in residual ridge remodeling and its clinical implications: a review. J Prosthet Dent 1998;80:224-237. DOI: 10.1016/S0022-3913(98)70116-7.

6. Cha H-S, Kim J-W, et al. Frequency of bone graft in implant surgery. Maxillofac Plast Reconstr Surg 2016;38:19. DOI: 10.1186/s40902-0160064-2.

7. Kumar P, Vanitha B, et al. Bone Grafts in Dentistry. J Pharm Bioallied Sci 2013;59(Suppl 1):S125-S127. DOI: 10.4103/0975-7406.113312.

8. Giannoudis PV, Dinopoulos $\mathrm{H}$, et al. Bone substitutes: an update. 2005;36(Suppl 3):S20-S27. DOI: 10.1016/j.injury.2005.07.029.

9. Kim A, Kar K, et al. Subapical osteotomy to correct dental implant malpositioning and vertical ridge deficiency: a critical report.J Prosth Dent 2012;108:204-208. DOI: 10.1016/S0022-3913(12)00135-7.

10. Sakkas A, Wilde F, etal. Autogenous bone grafts in oral implantology-is it still a "gold-standard"? a consecutive review of 279 patients with 456 clinical procedures. Int J Implant Dentistry 2017;3:23. DOI: 10.1186/ s40729-017-0084-4.

11. Sobhita G, Bobby J, et al. An overview of stem cell therapy in oral and maxillofacial surgery. Imp J Interdiscip Res 2017;3(10):504-509.

12. Masaru K, Yosuke A, et al. Cell-based bone regeneration for alveolar ridge augmentation-Cell source, endogenous cell recruitment and immunomodulatory function. J Prosthodon Res 2015;59:96-112. DOI: 10.1016/j.jpor.2015.02.001.

13. Meijer GJ, de Bruijn JD, et al. Cell based bone tissue engineering in jaw defects. Biomaterials 2008;29:3053-3061. DOI: 10.1016/ j.biomaterials.2008.03.012.

14. Shayesteh YS, Khojasteh A, et al. Sinus augmentation using human mesenchymal stem cells loaded into beta-tricalcium phosphate/ hydroxyapatite scaffold. Oral Surg Oral Med Pathol Oral Radiol Endod 2008;106:203-209. DOI: 10.1016/j.tripleo.2007.12.001.

15. McAllister BS, Haghighat K, et al. Histologic evaluation of a stemcell based sinus-augmentation procedure. J Periodontol 2009;80: 679-686. DOI: 10.1902/jop.2009.080345. 